\title{
SYNTHESIS OF BIO-STABILIZED GOLD NANO PARTICLE AND THEIR CHARACTERISATION
}

\author{
Alok Roy ${ }^{1}$, Bidhan Mohanta ${ }^{2}$ \\ ${ }^{1}$ Department of physics, Assam University, Silchar, Assam, 788011, India \\ ${ }^{2}$ Department of physics, Assam University, Silchar, Assam, 788011, India
}

\begin{abstract}
Gold nano particles were synthesised using Eupatorium Odoratum leaf extract. The method takes only few second for complete reduction and stabilization. Synthesized GNPs were characterized using UV-vis spectroscopy, Transmission Electron Microscopy, Fourier Transform Infra red spectroscopy, and X-ray diffractometer. The synthesized GNPs are well monodispersed with size ranging from 30-50 nm.FTIR spectra shows functional group attached with formed GNPS.XRD peak revealed the crystalline nature of synthesized GNPs.
\end{abstract}

\section{INTRODUCTION}

Gold nanoparticles are now a day has become most attracting research topic because of their highly tuneable optical property, biocompatibility, catalytic and less toxicityto the living cell[1,2]. These properties make them a suitable material for drug delivery, diagnosis devices and hyperthermia cancer therapies [3] Till date various synthesis techniques have been developed.Wet chemical synthesis methods to different physical method. Though in the wet chemical methods yielding of nanoparticles is very large but because of cytotoxity of synthetic reagents used like sodium borohydrate,cetrymethyle ammonium bromide (CTAB) it may not bea suitable one for bio analyticalapplication [4].Green synthesis of nano particle has overcome these problems by reducing hazardous and toxic bi-products and become an alternate of syntheticchemicals techniques. Asin greentechnique comprises of different plant parts, biomass and microorganisms etc that is all natural components so very less possibility of formation of toxic bi-products[5]. Among all the bio techniques plant leaf extract is wieldy explored as it is simple, one step, cost effective and ecofriendly and more over phythochemicals present in leaf extract act both as stabilising as well as capping agent [6,7]. Different plant extract like garlic [8], onion [9], lemongrass [10], neem [11] etc. have been used for synthesis of gold nano particles.

In this paper, we report a new synthesis procedure of gold nano particles using Eupatorium Odoratumleafextract. The plant leaf is a rich source of protein, biotin, ascorbic acid, steroids flavonoids and many others molecules of medicinal importance $[12,13]$.This procedure is rapid, facile, one step synthesis, moreover the synthesised gold nano particles are highly stable and monodisperse. The synthesised gold nano particles have characterised using uv-vis spectroscopy, TEM, FTIR spectroscopy, XRD.

\section{MATERIAL AND METHODS}

Tetra chloroauric acid $\left(\mathrm{HAuCl}_{4}\right)$ was purchased from SRL India. Fresh leaves were collected from village sides. Deionized water was used for all reaction and laboratory purpose.

\subsection{Preparation of Leaf Extract}

Leafextract were prepared by boiling dry leaves with deionised water. Extract is cooled to room temperature and kept for future uses.

\subsection{Synthesis of Gold Nanoparticles}

For gold nano particle synthesis, $10 \mathrm{ml}$ of $0.4 \mathrm{mM} \mathrm{HAuCl}_{4}$ solution was mixed with $10 \mathrm{ml}$ of DI water and heated for 15 minutes at $60^{\circ} \mathrm{C}$. Then $300 \mu \mathrm{l}$ of leaf extract was added to the above mixture and stirred moderately. Colour of the gold solution changed from yellowish to pink red within a minute. This is the primary indication of formation of gold nano particles.

\subsection{Characterization Techniques}

UV-Vis spectroscopy was performed using Perkin Elmer Lambda 25 to observe surface Plasmon resonance peak. Transmission electron microscopy (TEM) images were taken to confirm the nanoparticles formation and also to investigate their morphology using JEM-2100 TEM.Fourier transform infra red spectroscopy (FTIR) of the gold nanoparticles were taken using Perkin Elmer spectrum Two, to identify the functional groups associated with the synthesized GNP.XRD of GNP was carried out to check the crystalline nature of the prepared sample. 


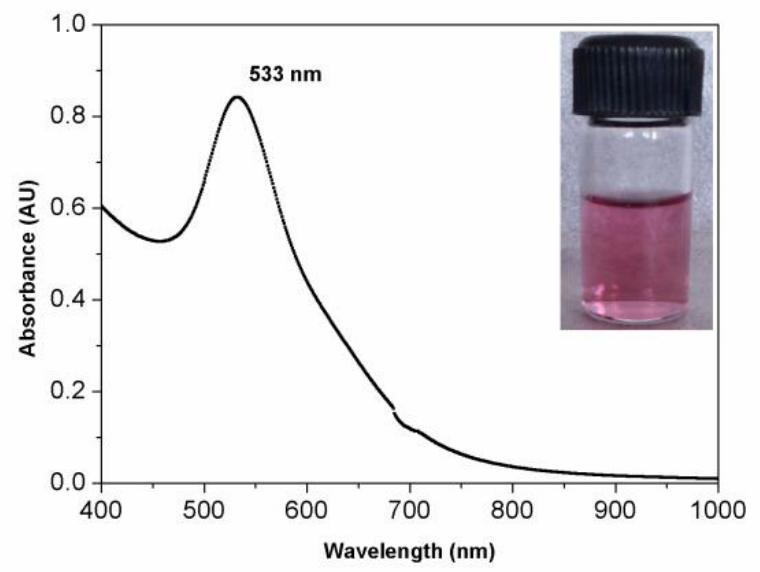

\section{RESULTS AND DISCUSSION}

Change of yellow colour of the cholroauric acid solution to reddish pink on addition of few drop of leaf extract is the primary indication of formation of gold nano particles. As different literature established the fact that colloidal gold solution shows intense colour in nano form [8].Itis further confirmed from uv-vis spectroscopic observation, which shows an intense observation at $533 \mathrm{~nm}$ (fig.1).It is a collective oscillation of free electrons if resonance with the oscillating electric field of light cause a strong absorption of energy. The sharp band at $533 \mathrm{~nm}$ also revealed that the synthesised particle are of isotropic shape as confirmed by transmission electron microscope (TEM) images fig.(2.a).with size distribution of $20 \mathrm{~nm}$ to

Fig. 1: UV-Vis spectrum of GNPs showing surface plasma resonance absorption peak at 533nm .Inset shows image of coloured GNPs formed.
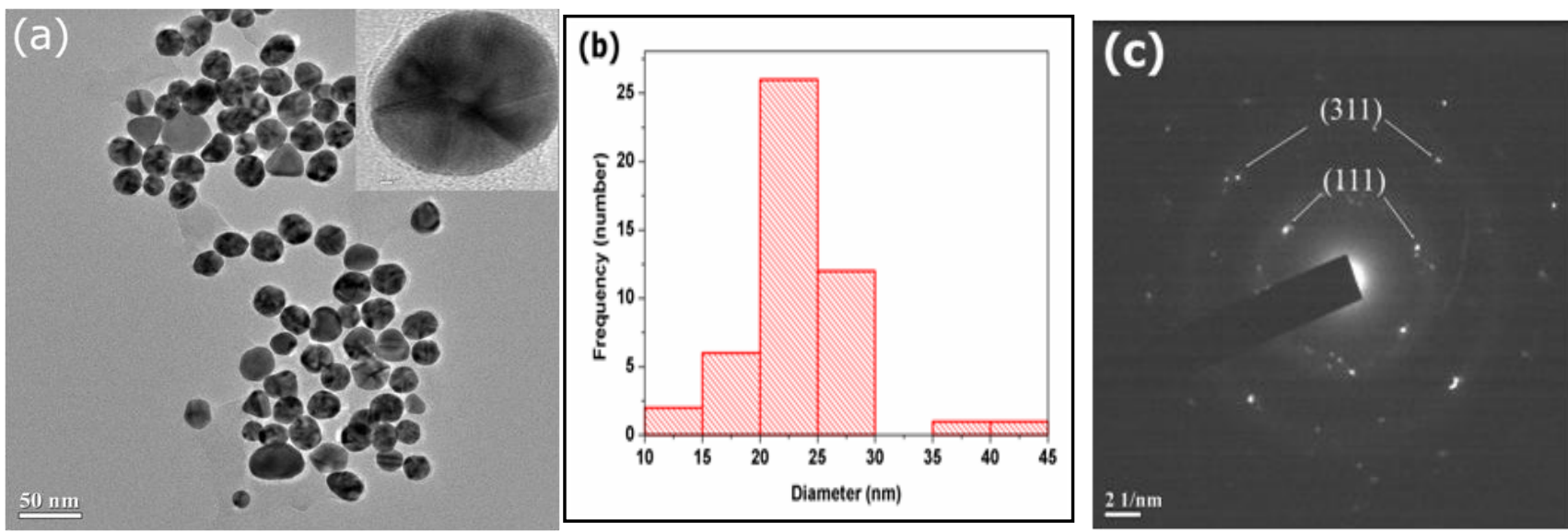

Fig.2: (a) TEM image of leaf extract synthesized GNPs, (inset) HRTEM micrographs showing typical morphologies of gold

$50 \mathrm{~nm}$ fig. (2.b).TEM images show that the synthesized nano particles are well separated from each other and of uniform shape and size, indicating that the stabilising agent, capping agent which are present in phythochemicals are very strong.HRTEM gives a close sight of spherical GNP.Selected area electron diffraction pattern (SAED) (fig.2.c) shows concentric bright ring corresponding to different crystal plane with plane index [111] and [311] indicating the FCC crystal structure corresponds to dspacing of $0.24 \mathrm{~nm}$ and $0.122 \mathrm{~nm}[14]$ this result is further verified from the XRD pattern of GNPs showing peaks corresponding to [111], [220],[311] planes.

The value $\mathrm{d}$-spacing between atoms is calculated from XRD data using Braggs equation $2 \mathrm{~d} \sin \theta=\mathrm{n} \lambda$, with $\lambda=1.54 \AA$.

\begin{tabular}{|l|l|l|l|}
\hline $\begin{array}{l}2 \theta(\text { Braggs } \\
\text { angle })\end{array}$ & $\Theta$ & $\begin{array}{l}\mathrm{d}-\text { spacing } \\
\mathrm{d}=\lambda / 2 \sin \\
(\mathrm{nm})\end{array}$ & $\begin{array}{l}\text { Reflection } \\
\text { plane }\end{array}$ \\
\hline 37.89 & 18.945 & 0.236 & {$[111]$} \\
\hline 64.065 & 32.035 & 0.145 & {$[220]$} \\
\hline 73.01 & 36.505 & 0.194 & {$[311]$} \\
\hline
\end{tabular}

Another point which is noticeable that we have used very little extract $1.5 \%$ of growth solution and it has been observed that the reaction rate is very rapid takes only a few minute,

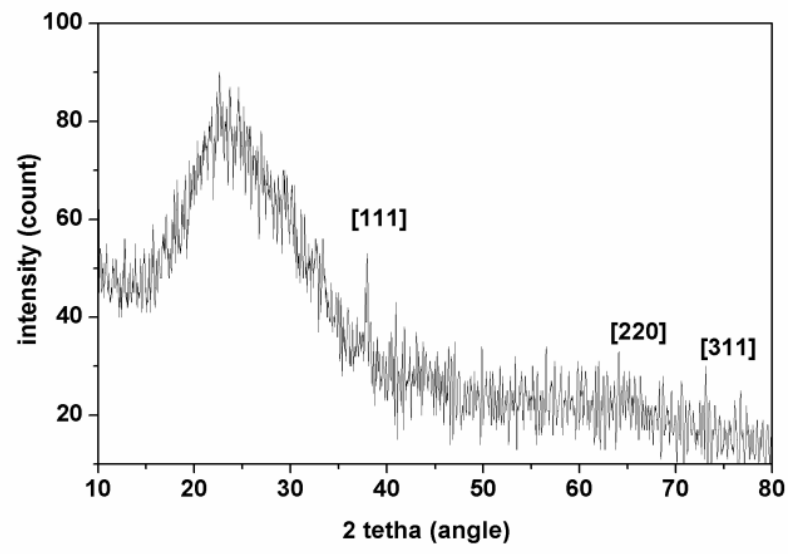

Fig.3: X-ray diffraction pattern of prepared gold nano particles.Labelled peaks corresponds to characteristic diffraction peaks of elementel gold. 
So it can be proposed that reducing agents of leaf extract is very strong. From the experimental investigation it has been observed that synthesis method is depending upon moderate heat. The optimum temperature which is favourable for the high yielding stable GNP formation is found to be lies between $60^{\circ} \mathrm{C}$ to $70^{\circ} \mathrm{C}$. (Fig.) As we have used bio molecule to synthesis GNP it is preferable to take FTIR spectroscopy in order to get theinformation about surface modification of gold nano particles. Dry solid form of leaf extract and GNP was mixed with $\mathrm{KBr}$ powder to perform FTIR.FTIR graph of both leaf extract and GNP shows almost similar absorption peak. A broad absorption at 3401 $\mathrm{cm}^{-1}$ and $3352 \mathrm{~cm}^{-1}$ for L.E and GNP due to hydrogen bonded $\mathrm{O}-\mathrm{H}$ bond present in alcohol.

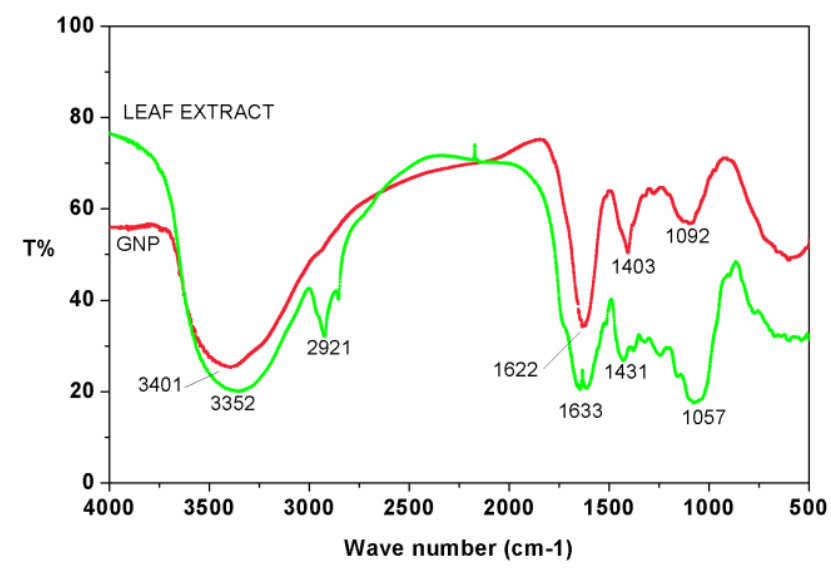

Fig.4: FTIR spectra of leaf extract and synthesized GNPs showing several absorption peaks corresponds to different functional group.

Few weak absorption near $2900 \mathrm{~cm}^{-1}$ only for LE corresponding to $\mathrm{C}-\mathrm{H}$ stretching vibration in methylene group. Vibration near $1633 \mathrm{~cm}^{-1}$ and $1622 \mathrm{~cm}^{-1}$ for the $\mathrm{C}=\mathrm{C}$ band. absorption near $1431 \mathrm{~cm}^{-1}$ and $1403 \mathrm{~cm}^{-1}$ indicating the presence of hydrocarbons of methylene group and vibration at $1057 \mathrm{~cm}^{-1}$ and $1092 \mathrm{~cm}^{-1}$ is corresponding to stretching CO vibration [15].FTIR spectra of both LE and GNPs showing similar absorption peak, only peak corresponds to $2900 \mathrm{~cm}^{-1}$ is disappear from the GNP spectrum indicating that functional group $\mathrm{C}-\mathrm{H}$ in methylene group involved as a capping agent of GNPs.

\section{CONCLUSION}

The method explored here to synthesise GNPs is rapid and yielding is high with high stability and monodispersed. The biochemical's presents in the leaf extract are act as reducing agent, capping agent. Moreover the method is free from any hazardous chemicals so it can be used as potent agent for bio-analytical application.

\section{ACKNOWLEDGMENT}

The authors would like acknowledge SAIF NEHU,Shillong for providing transmission electron microscopy facility and Manipur University for the XRD facility.

\section{REFERENCES}

[1] Xiaohua Huang, Mostafa A. El-Sayed . Journal of Advanced Research 1 (2010) 13-28.

[2] Madeeha A. Uppal, Michael B. Ewing, and Ivan P. Parkin. J. Inorg. Chem (2011) 4534-4544

[3] Panikkanvalappil R.Sajanlal, Theruvakkattil S.Sreeprasad, Akshaya K.Samal and Thalappil Pradeep. Nano Reviews 2 (2011) 5883.

[4] Nishima

Wangoo,K.K.Bhasin,S.K.Mehta,C.RamanSuri.Journa 1 of Colloid and Interface Science 323 (2008) 247254

[5] Sajid Fazal,Aswathy Jayasree, Sisini Sasidharan,Manzoor Koyakutty, Shantikumar V. Nair, and Deepthy Menon.ACS applied Materials \& Interfaces 2014, A-J.

[6] Rakhi Majumdar, Braja Gopal Bag and Nabasmita Maity, International Nano Letters 3 (2013) 53.

[7] Chelladurai Malarkodi, Shanmugam Rajeshkumar, Mahendran Vanaja, Kanniah Paulkumar,Gnanadhas Gnanajobitha and Gurusamy Annadurai.Journal Of Nanostructure in Chemistry 3 (2013) 30.

[8] Lori Rastogi, J Arunachalam. Adv Mat. Lett. 4 (7) (2013) 548-555.

[9] Umesh Kumar Parida,Birendra Kumar Bindhani, Padmalochan Nayak. World Journal of Nano Science and Engineering 1 ( 2011) 93-98.

[10] S. Shiv Shankar, Akhilesh Rai,Balaprasad Ankamwar, Amit Singh,Absar Ahmad and Murali Sastry. Nature Materials 3 (2004) 482-488.

[11] Priya Banerjee, Mantosh Satapathy, Aniruddha Mukhopahayay and Papita Das, Bioresources and Bioprocessing 1 (2014) 3

[12] Tamsyn Grewar,Mariekie Gericke. Advances in nanoparticles 1 (2012) 15-20.

[13] Jiale Huang,Qingbiao Li, Daohua Sun, Yinghua Lu Yuanbo Su, Xin Yang, Huixuan Wang, Yuanpeng Wang, Wenyao Shao, Ning He, Jinquing Hong and Cuixue. Chen. Nano technology 18 (2007) 105104 (11pp).

[14] Achintya Mohan Goswami, Sanjay Ghosh. Journal of Biomaterials and Nanobiotechnology 4 (2013) 20-27.

[15] Donald L. Pavia, Gary M. Lampman, George S. Kriz. (Thomson Learning, 2001) pp.13-101.

[16] Jude C. Ikewuchi; Catherine C. Ikewuchi, and Mercy O. Ifeanacho. The pacific Journal of Science and Technology 14(2) (2013) 360-378.

[17] Moses S. Owolabi, Akintayo Ogundajo, Kamil O. Yusuf, Labunmi Lajide, Heather E. Villanueva, Jessika A. Tuten, and William N. Setzer, Rec. Nat. Prod. 4(1) (2010) 72-78. 